C 2019 IEEE. Personal use of this material is permitted. Permission from IEEE must be obtained for all other uses, in any current or future media, including reprinting/republishing this material for advertising or promotional purposes, creating new collective works, for resale or redistribution to servers or lists, or reuse of any copyrighted component of this work in other works.

Paper Link: $\underline{\text { https://ieeexplore.ieee.org/document/8810658 }}$

Digital Object Identifier (DOI): 10.1109/PTC.2019.8810658

2019 IEEE Milan PowerTech, 23-27 June 2019.

\author{
A Method for Sizing Centralised Energy Storage Systems Using Standard Patterns \\ Shahab Karrari \\ Nicole Ludwig \\ Veit Hagenmeyer \\ Mathias Noe
}

Suggested Citation

S. Karrari, N. Ludwig, M. Noe, V. Hagenmeyer, "Sizing Centralized Energy Stor-age Systems in Distribution Networks Using Standard Patterns," in IEEE Power-Tech 2019, Milan, 2019. 


\title{
A Method for Sizing Centralised Energy Storage Systems Using Standard Patterns
}

\author{
Shahab Karrari, Nicole Ludwig, Veit Hagenmeyer, Mathias Noe \\ Karlsruhe Institute of Technology (KIT) \\ Karlsruhe, Germany \\ shahab.karrari@kit.edu
}

\begin{abstract}
Low Voltage (LV) distribution networks with high penetration levels of photovoltaics have to tackle various challenges such as overvoltages, voltage fluctuations, reverse power flows, and non-coincident demand and local generation. Energy Storage Systems (ESS) can help to ease these issues, if sized properly. This paper proposes a two-step methodology for sizing centralised ESS in LV networks. In the first step, a reoccurring daily pattern is detected using symbolic aggregated approximation (SAX) from the data measured at a German grid. Afterwards, high- and low-frequency components of the power signal are separated using a low-pass filter and then used for sizing different types of ESS. The effect of data resolution on the sizing outcomes is also investigated. The performance of the method was investigated using the full data set. It is concluded that ESS with the characteristics derived using this methodology can effectively be used for peak shaving, power smoothing and load balancing.
\end{abstract}

Index Terms-Energy Storage, Storage sizing, Batteries, Motif discovery.

\section{INTRODUCTION}

A high penetration level of photovoltaic (PV) production in low voltage (LV) distribution networks can lead to several challenges. For instance, the PV peak generation occurs in the middle of the day, when the demand is usually not peaking, especially in residential areas with a high full-time employment rate [1]. The generation without sufficient demand during midday can lead to a reverse power flow, which can lead to voltage-rise problems in parts of the LV network. This could be a limiting factor for the PV hosting capacity in LV networks. Furthermore, the variations in solar irradiance (e.g. caused by passing clouds) can cause rapid voltage fluctuations, which may violate the rapid voltage change thresholds, indicated in grid codes such as the EN 50160. Both voltage fluctuations and overvoltages occur due to the fact that $\mathrm{LV}$ distribution grids typically have relatively low $\mathrm{X} / \mathrm{R}$ ratios, which means that active power has a great influence on the voltage, as much as or even greater than reactive power. This can be shown using the voltage sensitivity formulation. Fig. 1 shows the singleline diagram of of a simplified grid structure. Between the point of common coupling (PCC) and the main supply, there is an equivalent resistance and reactance represented by $R_{\text {eq }}$

This work was supported by the German Research Foundation (DFG) as part of the Research Training Group GRK 2153: Energy Status Data Informatics Methods for its Collection, Analysis and Exploitation.

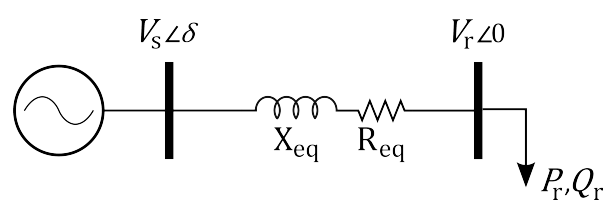

Fig. 1. Single-line diagram of an equivalent grid at the PCC.

and $\mathrm{X}_{\mathrm{eq}}$, respectively. By linearising the load flow equations around the operating point and assuming 1 p.u. for both $V_{\mathrm{s}}$ and $\mathrm{V}_{\mathrm{r}}$ voltages, the voltage sensitivity at the PCC can be calculated using [2]

$$
\begin{aligned}
\frac{\partial V_{\mathrm{r}}}{\partial P_{\mathrm{r}}} & =-\frac{\left(\mathrm{X}_{\mathrm{eq}}^{2}+\mathrm{R}_{\mathrm{eq}}^{2}\right) P_{\mathrm{r}}+\mathrm{R}_{\mathrm{eq}}}{1+2\left(\mathrm{R}_{\mathrm{eq}} P_{\mathrm{r}}+\mathrm{X}_{\mathrm{eq}} Q_{\mathrm{r}}\right)} \\
\frac{\partial V_{\mathrm{r}}}{\partial Q_{\mathrm{r}}} & =-\frac{\left(\mathrm{X}_{\mathrm{eq}}^{2}+\mathrm{R}_{\mathrm{eq}}^{2}\right) P_{\mathrm{r}}+\mathrm{X}_{\mathrm{eq}}}{1+2\left(\mathrm{R}_{\mathrm{eq}} P_{\mathrm{r}}+\mathrm{X}_{\mathrm{eq}} Q_{\mathrm{r}}\right)} .
\end{aligned}
$$

In addition, the evening demand peak coincides with a reduced or even non-existent PV generation. This requires ramping up of large conventional power plants. If many feeders in a power system behave in a similar manner, there might be a deficiency of available power plants with fast ramping capability.

Installing Energy Storage Systems (ESS) can help to overcome these challenges. Distribution networks can benefit from ESS for load balancing, peak shaving, voltage regulation, avoiding curtailment, energy arbitrage, loss reduction, expansion deferral and in other areas [3]. The usage of the properly sized ESS can also reduce reverse power flow and rapid voltage changes, which increases the dynamic and static PV hosting capacity of the grid. A centralised ESS, in comparison to distributed units, can be beneficial due to a more simplified control system, easy access to substation electrical and SCADA equipment, and availability utility-owned land. It can also be economical, as it only requires one grid interface and there is no need for a communication infrastructure. Nevertheless, the main question that needs to be addressed properly when installing an ESS in a power system is choosing the right capacity, rated power, most suitable technology and other characteristics of an ESS [4]-[6].

While there has been a significant amount of work on optimal sizing and allocation of distributed ESS, less attention 
has been paid to the sizing problem for centralised ESS. Comprehensive reviews of many of these methods can be found in [7] and [8]. For instance, a sizing method for distributed battery ESS for the purpose of voltage regulation and peak load shaving is introduced in [4]. ESS sizing algorithms can be based on the grid topology and its electrical characteristics or based on load and generation power profiles or a combination of both using sequential-time load flow. In the first approach, the optimal solution is often reached by performing $\mathrm{AC}$ or DC (or a hybrid) optimal power flow or by solving a nonlinear optimisation problem. These methods usually either do not use real load and generation data or assign artificial probabilistic quantities to them. In the second type of approaches, which is mostly used for centralised ESS, real load and generation data are used, as in this paper. In such studies, the time resolution of the data and the chosen time frame can play a significant role on the outcome of the study. While hourly data is usually used [4], [5], to fully investigate the benefits of using ESS in the presence of PV generation, resolution of $1 \mathrm{~s}$ may be required, in particular to cover fast changing generation, for instance caused by passing clouds over PV [9]. Authors in [10] propose a sizing strategy for a battery ESS with the purpose of peak shaving using 15-minute data. In this work, one arbitrary day is chosen for sizing, which can be misleading. In [11], a convex optimisation problem is proposed for the ESS sizing, for which the authors intentionally convert the 10s-data to 15-minute values to ease computations over a year, eliminating fast fluctuations of the power. In the same study, it has been shown that using a battery ESS to react immediately to mitigate effects of passing clouds, the lifetime of the battery diminishes over time. A similar approach is used in [5], but typical load profiles were generated with constant demands for each hour and in [12], 1-minute data are artificially generated from hourly data and the selection of a typical day is not clear.

In this paper, a two-step approach is introduced for sizing centralised ESS in LV distribution grids. In the first step, instead of using a an arbitrary day, a reoccurring daily pattern is detected with the help of a symbolic aggregated approximation (SAX) [13] of the time series. Real measurement data from a German LV substation with resolutions from 1 second to 10 minutes are used for this purpose. Different resolutions are used to investigate the effect of the data resolution on sizing results. In the next step, a Low-Pass Filter (LPF) designed with the help of Discrete Fourier Transform (DFT) is used to decompose fast and slow power variations of the daily pattern, which are then assigned to different types of ESS. ESS characteristics including nominal capacity, nominal power, maximum ramp rate and number of full cycles per day are derived from the data. Here, the main tasks of the ESS is assumed to be load balancing and power smoothing, which inherently leads to peak shaving, avoiding reverse power flow and reduction of voltage fluctuations. However, behindthe-meter applications of ESS such as increasing the selfconsumption can also be addressed using a similar approach.

The content of this paper is structured as follows. In section II, the methodology is explained in detail. A short discussion regarding the effect of the data resolution of sizing outcome is provided in part III. Afterwards, an evaluation of the proposed method is presented in part IV, followed by a conclusion and outlook in section $\mathrm{V}$.

\section{Methodology}

The first requirement for sizing ESS using time-series data is to select the power profile as the input for the sizing algorithm. To reduce computation time, it is a common practice to use an arbitrary day or a worst-case scenario data. However, in the present publication a SAX transformation and random projection are used to detect and generate daily patterns with the highest probability. In the next step, a LPF allocates different power profiles to different types of ESS. The method is discussed in detail in the following.

\section{A. Getting Standard Patterns}

Sizing of a ESS could be done by using the raw measured data, however, this approach can lead to a very data specific storage size. With only limited data available this might not be suitable, additionally with a large amount of data this approach can be computationally intensive. For this purpose, we want to find a standard consumption pattern and size our storage with regard to this standard pattern. The approximation of the whole time series to a standard pattern helps us to focus on regular shapes of the consumption instead of single occurrences. Two weeks of measurement data with the resolution of 1 second, 1 minute and 10 minutes from a German MV/LV substation is used for this purpose. The measured 1-second data for a period of two weeks is shown in Fig. 2(a).

Finding patterns in energy time series is a common unsupervised learning problem. In this paper, the problem is tackled using a motif discovery algorithm following [14] and [15]. For this motif discovery algorithm, the time series is first described with symbols and the random projection algorithm is used to find possible motifs. With the help of a sliding window a matrix $S^{*} \in(n-m+1) \times w$ is generated, with $n$ being the number of observations, $m$ the number of sequences and $w$ the predefined length of the motif. $w$ is chosen to cover a full day, for instance, $w=86400$ for the 1 second data. Each piece in a sequence is attributed a letter out of an alphabet. Different to the above mentioned implementations, the alphabet distribution is based on the empirical cumulative distribution function of the time series, which is defined as follows. For observations $x=\left(x_{1}, x_{2}, \ldots, x_{n}\right), F_{n}$ is the fraction of observations less or equal to $p$, i.e.,

$$
F_{n}(p)=\frac{1}{n} \sum_{i=1}^{n} \mathrm{I}\left(x_{i} \leq p\right) .
$$

The SAX representation of all sequences, are saved rowwise in a similarity matrix. In every iteration of the random projection algorithm, we randomly select $l$ of the $w$ columns of $S^{*}$, where $l$ is a user-defined mask length and $l \leq w$. The word built with $l$ columns is compared to all $(n-m+1)$ rows of $S^{*}$. If there exists a match between the letters in the mask, the corresponding entry in the so-called collision 


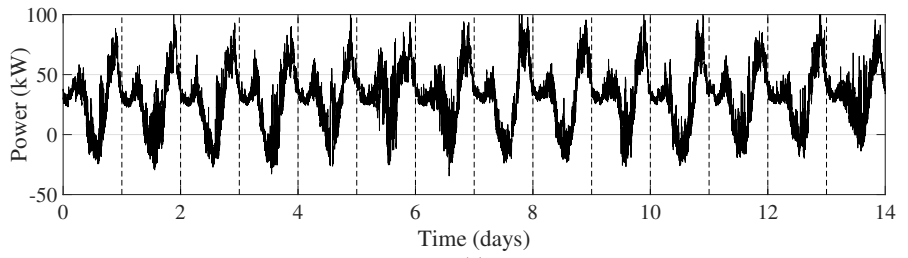

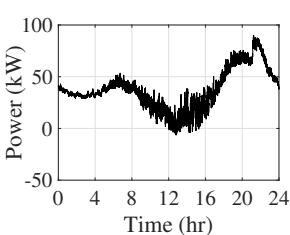

(a)

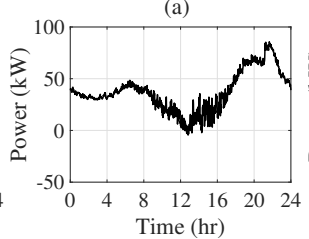

(c)

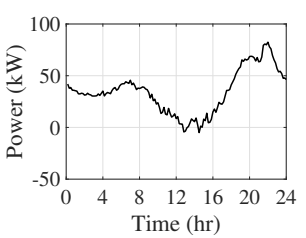

(d)
Fig. 2. (a) Measured power at the substation for two weeks with 1-second resolution. (b) $80 \%$-quantile (daily pattern) of 1-second data. (c) $80 \%$-quantile of the 1-minute data. (d) $80 \%$-quantile of the 10-minute data.

matrix is incremented. The entries with the highest values in the collision matrix are considered potential motifs. Those motifs are then iterated over the original time series and their distance is calculated to find the instances where the motif occurs. In the present paper, the motif candidates found are evaluated using the Euclidean distance between two time series sequences $s_{i}$ and $s_{j}$, which is defined as

$$
d\left(s_{i}, s_{j}\right)=\sqrt{\sum_{t}\left(s_{i}(t)-s_{j}(t)\right)^{2}} .
$$

The resulting motif covers most of the days in the data set. All these days grouped into the motif are used to describe the standard pattern. This standard pattern is then defined as the $80 \%$-quantile of all the occurrences. This quantile is chosen as the storage does not need to cover the maximum consumption which has ever happened, as the LV costumers are also supported by the grid. However, choosing a higher percentage quantile can easily be done, which will lead to bigger storage units.

The resulting standard pattern are shown in Fig. 2(b)-(d). As seen, the daily consumption pattern is a typical duck curve, which exhibits characteristics such as reverse power flow, high ramping and peak requirements and also significant power fluctuations during midday. These power profiles are used as the input for the second part of the sizing algorithm.

\section{B. Time-frequency Analysis}

Different ESS technologies operate at different time scales. While batteries have relatively large energy density, deep and frequent cycles can effect their lifetime and capacity. On the other hand, fast ESS such as supercapacitors or flywheel have high power density and can provide a large number of cycles without degradation, but their energy content is limited.

Therefore, a frequency analysis of the power profile can be a good reference point for ESS sizing. Using Discrete Fourier transform (DFT), it can found how much of the power signal lies within each given frequency band. Fig. 2(a) shows the frequency spectrum of the power profile for the three

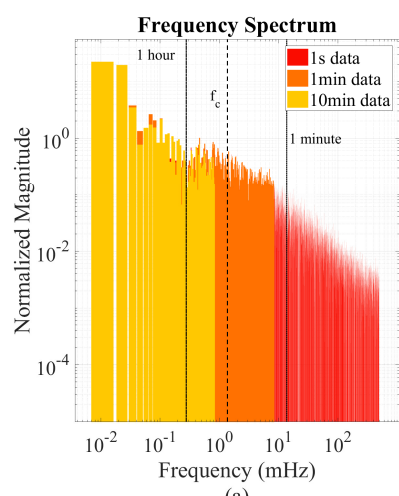

(a)

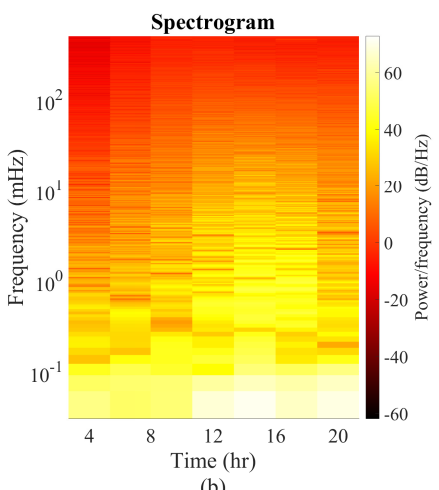

(b)
Fig. 3. (a) Frequency spectrum of the power profile at different resolutions. (b) Spectrogram of the $1 \mathrm{~s}$ daily pattern.

different time resolution. The spectrum starts at $0.01157 \mathrm{mHz}$ (corresponds to 24 hours) and ends at half of the sampling frequency, as according to the Nyquist principle the spectrum is symmetrical around half of the sampling frequency. As a non-periodic signal, the frequency spectrum of the power profile might be less visually interpretable, compared to a periodic signal. However, one can easy see frequencies below $13.793 \mathrm{mHz}$, which corresponds to 1 minute and $0.278 \mathrm{mHz}$, which corresponds to 1 hour (shown by vertical lines in Fig. 2 (a)). The effect of eliminating high-frequency components by using low-resolution data on the sizing outcomes are later discussed in section III. Fig. 3(b) shows the spectrogram of the 1 second signal using short-time DFT for every 3 hours using a Hamming window and a $50 \%$ overlap. This helps to visualise the time at which various frequencies are present. As expected, the higher frequencies are mostly present in the period starting at noon with the increase in PV production.

\section{Allocating Power Profiles}

Using a LPF to allocate different power signals to different ESS technology is a common practice, in particular for sizing battery-supercapacitor hybrid ESS [16]. In such systems, it has been shown that a smoother power profile with the help of supercapacitors significantly improves the lifetime of the batteries [17]. Here, the output of the LPF is used for sizing an electrochemical ESS such as Lithium-ion (Li-ion) batteries, which we refer to as type $1 \mathrm{ESS}$. The high-frequency components can either be provided by the grid or preferably by a fast ESS such as a supercapacitor or a flywheel, which is referred to as type $2 \mathrm{ESS}$. The cut-off frequency of the LPF influences the sizing results. However, despite many efforts there is no clear and agreed-upon method for selecting the cutoff frequency. In this work, the cut-off frequency is selected according to a typical discharge time of type 2 ESS. A LPF with a cut-off frequency of $1.33 \mathrm{mHz}\left(\mathrm{f}_{\mathrm{c}}\right.$ in Fig. 3), which corresponds to 75 seconds, is selected in this study. It is assumed that the average power over a day can be provided by the grid, as an autonomous operation of the distribution grid is not intended. Fig. 4 shows the power profile used for 


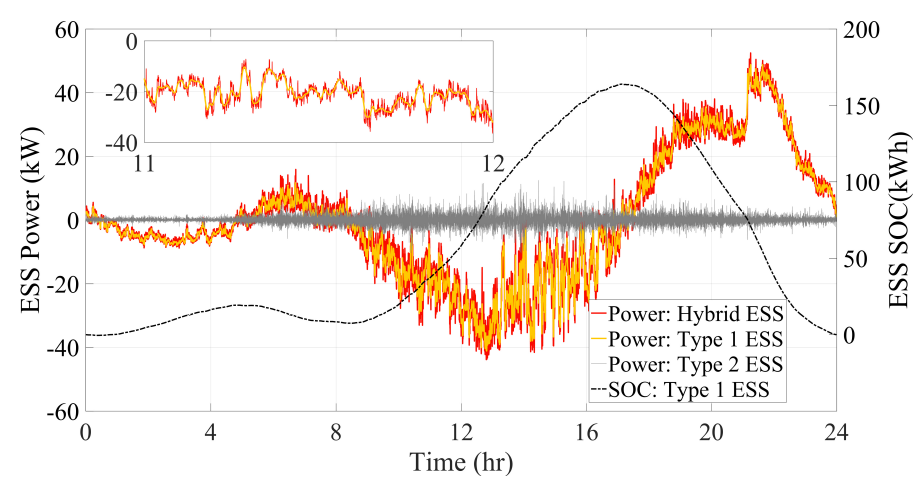

Fig. 4. Power of both types of ESS and SOC of type 1 ESS generated from the daily pattern.

sizing each type of ESS and their combination and how the State of Charge (SOC) of type 1 ESS changes over the daily pattern.

The method discussed here can also be used for sizing other Distributed Energy Resources (DER) such as fuel cells or diesel generators in combination with a type 2 ESS. In this case, the fast ESS allows a more efficient operation of the DER and a more economic sizing [12]. However, in this case, the negative power of the DER should be covered in another way, as it cannot consume energy.

\section{Deriving Energy Storage Characteristics}

In the following, the power profiles derived from the previous section is used for the sizing procedure. The sizing of the ESS is carried out using data with three different resolutions to see the effect of the data resolution on the sizing outcome. Furthermore, in addition to the nominal capacity of the ESS, other characteristics of the ESS such as nominal power, ramp rate, and cycling times per day is derived from the data. Here, for both charging and discharging mode of the ESS, an efficiency of $90 \%$ is considered.

a) Nominal capacity: The nominal capacity of an ESS depends on the storage medium itself, which in case of Liion batteries depends on the number of cells and in case of flywheels, depends on the inertia and the maximum speed of the rotating mass. The nominal capacity $\left(E_{n}\right)$ for each type of the ESS can simply be calculated by integrating over the power allocated to each one of them and selecting its maximum, i.e.

$$
\mathrm{E}_{\mathrm{n}}=\frac{\left.\max \left(\left|\int_{0}^{t} P(\tau) d \tau\right|\right)\right)}{\eta\left(1-\frac{\mathrm{SOC}_{\min }}{\mathrm{SOC}_{\max }}\right)} .
$$

where $P(\tau)$ is the power allocated to the ESS and $\eta$ is its efficiency. For Li-ion batteries, the lifetime can further be extended by operating it within a certain range of SOC [11], here represented by $\left[\mathrm{SOC}_{\min }, \mathrm{SOC}_{\max }\right]$. Therefore, this range is also taken into when sizing the ESS.

b) Nominal power: The nominal power $\left(\mathrm{P}_{\mathrm{n}}\right)$ of the ESS is commonly limited by the power electronics interface of the ESS. Thus, increasing power ratings is generally less expensive than increasing the energy ratings. For both types of ESS, the maximum of the assigned power is chosen, considering both charging and discharging, i.e.

$$
\mathrm{P}_{\mathrm{n}}=\max (\max (P(t)),|\min (P(t))|) .
$$

c) Ramp rate: In the literature, there are various definitions for a ramp rate. In this paper, the ramp rate $\left(R_{n}\right)$ is considered as the maximum change of power between two consecutive points in time. If $\mathrm{T}_{\mathrm{S}}$ is the sampling time of the power signal, the ramp rat is calculated as

$$
\mathrm{R}_{\mathrm{n}}=\max \left(P(t)-P\left(t-\mathrm{T}_{\mathrm{s}}\right)\right) .
$$

d) Cycling times and lifetime: Cycling times is defined here as the number of times the SOC of the ESS falls below $10 \%$ of its maximum, which is limited value for each technology. In electrochemical batteries such as Li-ion batteries, the lifetime deteriorates with deep cycling, due to cumulative changes of the structure and decomposition of the cells. For such batteries the following charging/discharging behaviour can significantly affect its lifetime [11]:

1) High rates of charging/discharging.

2) Frequent variation in the rate of charge/discharge.

3) Leaving the battery for a long time at high SOC.

4) Cycling more than once a day. This simply reduces the calendar life of the battery.

The first two factors can be avoided by allocating the high-frequency components to a type 2 ESS or the grid, as suggested earlier. The third point can be taken into account by choosing a larger battery, as shown in Eq. (5). The last influencing factor depends on the charging and discharging algorithm and how often the batteries operation is triggered. Thereby, the control algorithm should only allows a battery to be charged and discharged once during a day. This is later discussed in section IV.

\section{E. Choosing the right technology}

The aforementioned characteristics for the two types of ESS are calculated and presented in TABLE I. In each case, the ESS should have the sufficient capacity, power, ramp rate, and cycling times. For type 1 ESS, an ESS with high energy content is required with energy to power ratio of approximately three. This can easily be provided by Li-ion batteries. Also, redox flow battery can be used, as the energy and power of flow batteries can be scaled independently from each other. For type 2 ESS Flywheel Energy Storage System (FESS) and supercapacitors are a good alternative. Flywheel Energy Storage Systems (FESS) were proposed as an optimal solution for power smoothing or other applications, where frequent cycling at high powers are required [18], [19].

\section{DISCUSSION ON TIME RESOLUTION OF DATA}

In the present paper, the sizing calculations are carried out using 1-second, 1-minute and 10-minute data. As shown in TABLE I, there is no significant difference between the sizing results for type 1 ESS, when using 1-minute data and 1-second 
TABLE I

ESS CHARACTERISTICS DERIVED FROM THE DATA.

\begin{tabular}{|l|c|c|c|c|c|}
\hline Type of ESS & \multicolumn{3}{|c|}{ Type 1 ESS } & \multicolumn{2}{c|}{ Type 2 ESS } \\
\hline Data Resolution & $1 \mathrm{~s}$ & $1 \mathrm{~min}$ & $10 \mathrm{~min}$ & $1 \mathrm{~s}$ & $1 \mathrm{~min}$ \\
\hline Nom. Capacity (kWh) & 163.86 & 162.71 & 171.9 & 0.054 & 2.2 \\
Nom. Power (kW) & 50.32 & 44.75 & 48.69 & 12.89 & 21.27 \\
Ramp Rate (kW/s) & 0.54 & 0.42 & 0.017 & 13.93 & 0.27 \\
Full cycles per day & 1 & 1 & 1 & 29 & 4 \\
\hline Suitable Technology & \multicolumn{3}{|l|}{ Li-ion batteries, } & \multicolumn{3}{c|}{ Flywhels, } \\
\end{tabular}

data. Therefore, it can be concluded that using 1 minute data is sufficient for sizing ESS for applications such as peak shaving and load balancing. Using 10-minute data leads to a slightly bigger storage, however, the required ramp rate cannot be accurately calculated. For power smoothing applications using type 2 ESS, 1-second data is the most advantageous, as these system often operate within a few seconds. Even using 1minute data can lead to oversizing and and underestimating the ramp rate.

\section{Evaluation}

For the evaluation of the proposed sizing methodology, the derived ESS characteristics are tested with the two weeks measurement data. As the $80 \%$ quantile is chosen for the sizing, it is clear that the ESS cannot cover the power variations at all times. However, for the intended purposes of peak shaving, load levelling and power smoothing, the effects of the ESS can be assessed. To fully observe the effect of power smoothing by the type 2 ESS, the evaluation results are presented using the 1-second data and its corresponding ESS characteristics. However, similar results can be presented using the 1-minute and 10-minute data. The results are obtained using the constraints presented in TABLE I, meaning that energy content, power, and ramping rate and cycling times of each ESS are kept below calculated values. For the peak shaving implementation, a simple controller triggers the type 1 ESS to store energy from 11:00 to 16:00 and discharges it from 18:00 to 00:00. These times are also selected based on the detected daily pattern (see Fig. 2(b)). For type 2 ESS, a continuous operation is assumed.

In Fig. 5(a), four different cases are compared to each other. Case 1 shows the originally measured consumption at the substation. Case 2 and case 3 show the power drawn from the grid if only one type of ESS, type 1 or type 2, is installed at the substation. Case 1 and case 3 are hard to distinguish, however case 3 has a much smoother curve, as shown in Fig. 5(d). Finally, the ideal case, in which both ESS are installed, either separately or in a hybrid structure, is presented as case 4 . By analysing the results in Fig. 5, the following observations can be made:

1) As seen in Fig. 5(a), by using type 1 ESS, the evening peak is eliminated for almost everyday. That is a reduction of $45.32 \mathrm{~kW}$ on average. The exception is the $6^{\text {th }}$ day, in which there was no sufficient PV generation to charge the type 1 ESS and cover the peak demand. Due to the peak shaving, the grid does not need to provide the power with high ramp rate in the afternoon. Moreover, the ESS can defer expansions or relief congestion from the grid components, if that is the case. The results for the $5^{\text {th }}$ day are presented separately in Fig. 5(c).

2) During midday, reverse power flow is either avoided or its duration and power is significantly reduced. This can potentially increase the static hosting capacity of the LV grid for PV installations and avoids PV curtailment due to overvoltage issues. However, if a PV system is installed far away from the storage installation place, it may still require local voltage compensation.

3) As shown in Fig. 5(d), type 2 ESS reduces shorttime power fluctuations. The maximum change in power during the whole period is reduced by $35.2 \%$, from 18.8 $\mathrm{kW} / \mathrm{s}$ to $12.2 \mathrm{~kW} / \mathrm{s}$, while on average it is down to $54.38 \%$. This will increase the dynamic hosting capacity of the LV grid, as the number of rapid voltage changes due to the active power variations, such as the ones caused by passing clouds, are reduced. Moreover, in case both ESS are in operation, the power drawn from the grid is as smooth and flat as possible, taking into account the ESS constraints, such as their ramp rate.

4) By using type 2 ESS, the power drawn by or injected by type 1 ESS has significantly less variations, which can improve the lifetime of such systems significantly.

\section{CONCLUSION}

In the present paper, a new approach for sizing centralised ESS is introduced. A reoccurring daily pattern with high probability is first detected and extracted using a SAX transformation and random projection. This pattern is then broken down to high- and low-frequency components for sizing two types of ESS with specific applications. For each type of ESS, nominal capacity, nominal power, ramp rate, number of cycles per day and most suitable technology is derived from the data. In addition, the effects of using measurement data with different resolutions on the sizing outcome is investigated. It is concluded that for applications such as peak shaving, using 1 minute sampled data is still adequate, while using 10-minute sampled data can lead to minor oversizing and an inaccurate ramp rate. However, using 1 second data for power smoothing applications is mandatory. Finally, the sizing outcomes are then evaluated using 14 days of data. It has been shown that ESS with characteristics derived from detected daily consumption pattern, can effectively reduce peak demand, reverse power flow and voltage fluctuations over the whole period.

For future work, a cost-benefit analysis can complement the proposed method. Other possible influencing factors in the sizing of the ESS, such as a more detailed modelling of the ESS and it power flow controller can be added to the methodology. 


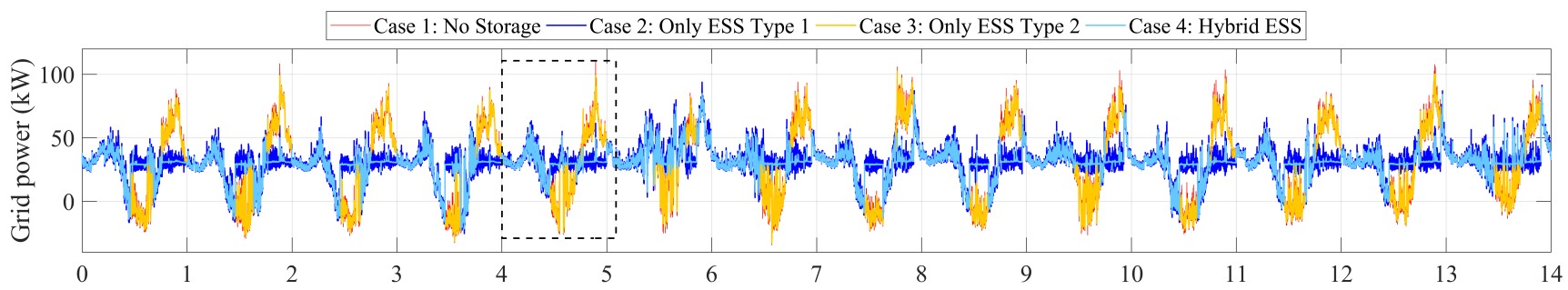

(a)

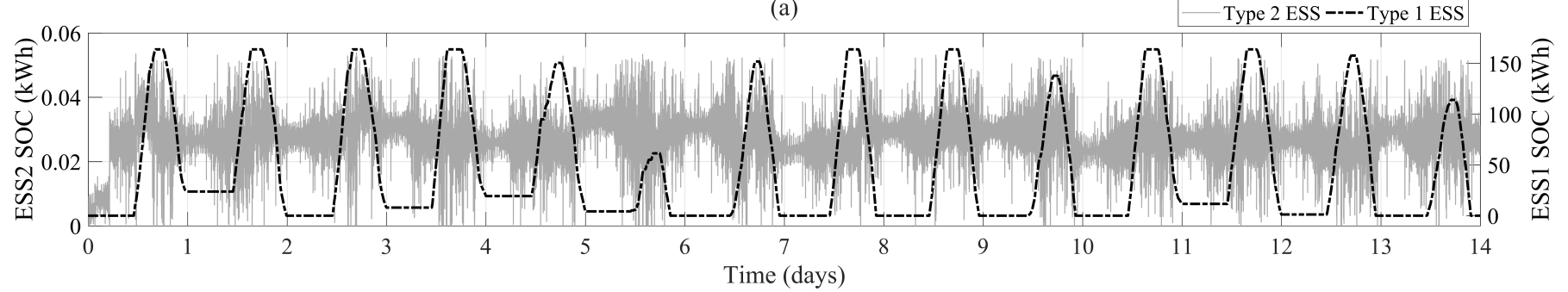

(b)

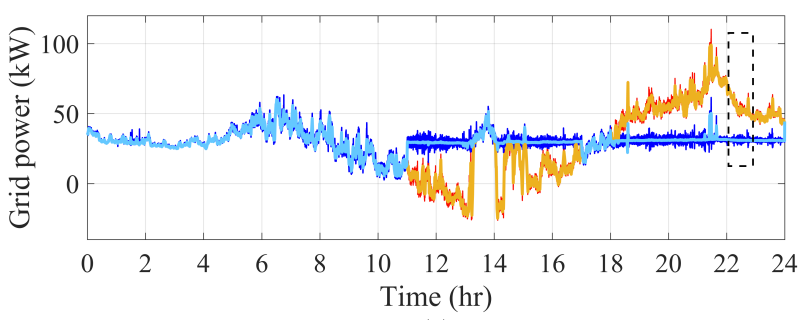

(c)

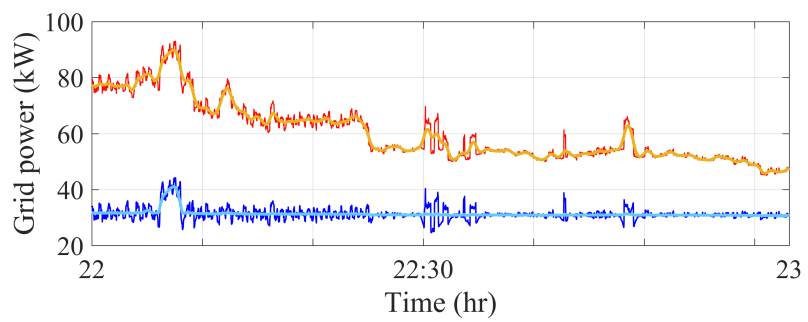

(d)

Fig. 5. (a) Power drawn from the grid in different scenarios during two weeks. (b) SOC of type 1 and type 2 ESS during two weeks. (c) Power drawn from the grid during the $5^{t h}$ day. (d) Power drawn from the grid from 22:00 to 23:00 during the $5^{t h}$ day.

\section{REFERENCES}

[1] M. Hayn, V. Bertsch, and W. Fichtner, "Electricity load profiles in Europe: The importance of household segmentation," Energy Research and Social Science, vol. 3, no. C, pp. 30-45, 2014.

[2] Y. Hase, Handbook of Power Systems Engineering with Power Electronics Applications, 2nd ed. John Wiley \& Sons, 2013.

[3] "European energy Storage Technology Development Roadmap - 2017 Update," EASE/EERA, Tech. Rep., 2017.

[4] Y. Yang, H. Li, A. Aichhorn, J. Zheng, and M. Greenleaf, "Sizing strategy of distributed battery storage system with high penetration of photovoltaic for voltage regulation and peak load shaving," IEEE Transactions on Smart Grid, vol. 5, no. 2, pp. 982-991, 2014.

[5] Y. Ru, J. Kleissl, and S. Martinez, "Storage size determination for grid-connected photovoltaic systems," IEEE Transactions on Sustainable Energy, vol. 4, no. 1, pp. 68-81, 2013.

[6] Y. V. Makarov, P. Du, M. C. W. Kintner-Meyer, C. Jin, and H. F. Illian, "Sizing energy storage to accommodate high penetration of variable energy resources," IEEE Transactions on Sustainable Energy, vol. 3, no. 1, pp. 34-40, 2012.

[7] N. D. Hatziargyriou, D. Škrlec, T. Capuder, P. S. Georgilakis, and M. Zidar, "Review of energy storage allocation in power distribution networks: applications, methods and future research," IET Generation, Transmission \& Distribution, vol. 10, no. 3, pp. 645-652, 2016.

[8] A. Chauhan and R. P. Saini, "A review on Integrated Renewable Energy System based power generation for stand-alone applications: Configurations, storage options, sizing methodologies and control," Renewable and Sustainable Energy Reviews, vol. 38, pp. 99-120, 2014.

[9] R. C. Dugan, J. A. Taylor, and D. Montenegro, "Energy Storage Modeling for Distribution Planning," IEEE Transactions on Industrial Applications, vol. 53, no. 2, pp. 954-962, 2017.

[10] A. Oudalov, R. Cherkaoui, and A. Beguin, "Sizing and optimal operation of battery energy storage system for peak shaving application," 2007 IEEE Lausanne PowerTech, pp. 621-625, 2007.

[11] A. Nagarajan and R. Ayyanar, "Design and Strategy for the Deployment of Energy Storage Systems in a Distribution Feeder With Penetration of Renewable Resources," IEEE Transactions on Sustainable Energy, vol. 6, no. 3, pp. 1085-1092, 2015.

[12] J. Xiao, L. Bai, F. Li, H. Liang, and C. Wang, "Sizing of energy storage and diesel generators in an isolated microgrid Using Discrete Fourier Transform (DFT)," IEEE Transactions on Sustainable Energy, vol. 5, no. 3, pp. 907-916, 2014.

[13] J. Lin, E. Keogh, L. Wei, and S. Lonardi, "Experiencing SAX: A novel symbolic representation of time series," Data Mining and Knowledge Discovery, vol. 15, no. 2, pp. 107-144, 2007.

[14] B. Chiu, E. Keogh, and S. Lonardi, "Probabilistic discovery of time series motifs," in Proceedings of the ninth ACM SIGKDD international conference on Knowledge discovery and data mining - KDD '03, 2003, pp. 493-498.

[15] L. Barth, V. Hagenmeyer, N. Ludwig, and D. Wagner, "How Much Demand Side Flexibility Do We Need?: Analyzing Where to Exploit Flexibility in Industrial Processes," in Proceedings of the Ninth International Conference on Future Energy Systems, Karlsruhe, 2018, pp. 43-62.

[16] D. B. W. Abeywardana, B. Hredzak, V. G. Agelidis, and G. D. Demetriades, "Supercapacitor sizing method for energy-controlled filterbased hybrid energy storage systems," IEEE Transactions on Power Electronics, vol. 32, no. 2, pp. 1626-1637, 2017.

[17] A. M. Gee, F. V. P. Robinson, and R. W. Dunn, "Analysis of Battery Lifetime Extension in a Small-ScaleWind-Energy System Using Supercapacitors," IEEE Transactions on Energy Conversion, vol. 28, no. 1, pp. 24-33, 2013.

[18] C. S. Hearn, M. C. Lewis, S. B. Pratap, R. E. Hebner, F. M. Uriarte, D. Chen, and R. G. Longoria, "Utilization of optimal control law to size grid-level flywheel energy storage," IEEE Transactions on Sustainable Energy, vol. 4, no. 3, pp. 611-618, 2013.

[19] S. Karrari, M. Noe, and J. Geisbuesch, "High-speed Flywheel Energy Storage System (FESS) for Voltage and Frequency Support in Low Voltage Distribution Networks," in 2018 IEEE 3rd International Conference on Intelligent Energy and Power Systems (IEPS), 2018, pp. 176-182. 